\title{
Efeito do exercício prévio severo nos parâmetros da relação po- tência - tempo em indivíduos ativos no ciclismo
}

\author{
Effect of previous severe exercise on the power-time relationship in ac- \\ tive individuals during cycling
}
Renato Aparecido Corrêa Caritá ${ }^{1}$, Cintia Picarelli ${ }^{1}$, Camila Coelho Greco ${ }^{1 *}$, Benedito Sér- gio Denadai ${ }^{1}$

ARTIGO ORIGINAL | ORIGINALARTICLE

O objetivo deste estudo foi analisar o efeito do exercício prévio severo (EPS) sobre a potência crítica (PC) e a capacidade de trabalho anaeróbio (CTA) determinados durante o exercício de ciclismo. Sete indivíduos realizaram os seguintes protocolos em cicloergômetro: (a) teste progressivo para determinação do limiar ventilatório $(\mathrm{LV})$, consumo máximo de oxigênio $\left(\mathrm{VO}_{2} \mathrm{max}\right)$ e sua respetiva intensidade $\left(\mathrm{IVO}_{2} \mathrm{max}\right)$; (b) seis testes de carga constante em ordem aleatória, com (EPS) e sem (CON) a realização de EPS. O EPS foi realizado durante $6 \mathrm{~min}$ a $70 \% \Delta$ (i.e., $70 \%$ da diferença entre o LV e a $\left.\mathrm{IVO}_{2} \mathrm{max}\right)$. A PC na condição CON $(199.71 \pm 27.08 \mathrm{~W})$ não foi significativamente diferente da condição EPS $(211.71 \pm 29.92 \mathrm{~W})$. Do mesmo modo, a CTA não foi significativamente diferente entre as condições $(\mathrm{CON}=23.46 \pm 10.76 \mathrm{~kJ}$ vs. EPS $=17.51 \pm 8.44 \mathrm{~kJ})$. Portanto, o exercício prévio severo $(70 \% \Delta)$ seguido por 6 min. de recuperação não influencia a determinação da PC e da CTA em indivíduos ativos.

Palavras-chaves: treinamento aeróbio, aquecimento, metabolismo

ABSTRACT

The aim of this study was to analyse the effect of prior severe exercise (PSE) on the critical power (CP) and anaerobic work capacity (AWC) determined during cycling exercise. Seven males, performed on different days, the following protocols on a cycle ergometer: (a) incremental test to determine ventilatory threshold (VT), maximal oxygen consumption $\left(\mathrm{VO}_{2} \mathrm{max}\right)$ and its corresponding intensity $\left(\mathrm{IVO}_{2} \mathrm{max}\right)$, (b) six constant-work-rate tests in random order, with (PSE) and without (CON) the completion of PSE. The PSE was preceded by $6 \mathrm{~min}$ at $70 \% \Delta$ (i.e., $70 \%$ of the difference between VT and $\left.\mathrm{IVO}_{2} \mathrm{max}\right)$. The estimation of the $\mathrm{CP}$ at $\mathrm{CON}$ condition $(199.71 \pm 27.08 \mathrm{~W})$ was not significantly different from the condition PSE $(211.71 \pm 29.92 \mathrm{~W})$. Similarly, AWC was not significantly different between conditions $(\mathrm{CON}=23.46 \pm 10.76 \mathrm{~kJ}$ vs. $\mathrm{PSE}=17.51 \pm 8.44 \mathrm{~kJ})$. Thus, prior severe exercise $(70 \% \Delta)$ followed by 6 min of recovery does not influence the estimation of CP and AWC in active individuals.

Keywords: aerobic training, warm up, metabolism

Artigo recebido a 09.09.2013; Aceite 02.06.2014

${ }^{1}$ Laboratório de Avaliação da Performance Humana - UNESP - Rio Claro, São Paulo, Brasil

* Autor correspondente: Av. 24 A, 1515, Bela Vista - Rio Claro - SP - Brasil - CEP - 13506-900;

E-mail: grecocc@rc.unesp.br 


\section{INTRODUÇÃO}

As respostas do consumo de oxigênio $\left(\mathrm{VO}_{2}\right)$ e do lactato sanguíneo ao exercício de carga constante têm sido utilizadas para caracterizar os diferentes domínios de intensidade de exercício (i.e., moderado, pesado e severo) (Gaesser \& Poole, 1996). No domínio moderado, as concentrações de lactato são próximas aos valores de repouso e o $\mathrm{VO}_{2}$, após 2-3 minutos do início do exercício, apresenta-se estável ao longo do tempo. Assim, este domínio tem seu limite superior no limiar de lactato (LL - primeiro e sustentado aumento da concentração de lactato em relação aos valores de repouso). O domínio pesado apresenta intensidades de exercício entre o LL e a potência crítica (PC). Estas intensidades apresentam concentrações mais elevadas de lactato sanguíneo $(\sim 2-6$ $\mathrm{mM})$, mas que ainda possuem estabilidade ao longo do tempo. Neste domínio, pelo surgimento da componente lenta, o $\mathrm{VO}_{2}$ apresenta uma estabilidade mais tardia (i.e., após 15-20 min). No domínio severo, que compreende intensidades de exercício acima da PC, o lactato sanguíneo e o $\mathrm{VO}_{2}$ não apresentam estabilidade ao longo do tempo. A maior amplitude da componente lenta do $\mathrm{VO}_{2}$ permite que seu valor máximo (i.e, $\mathrm{VO}_{2} \max$ ) seja alcançado.

Para exercícios realizados no domínio severo, a relação entre a potência gerada externamente $(\mathrm{P})$ e o seu respetivo tempo máximo de exercício (i.e., tempo limite - tlim) tem sido descrita por diferentes modelos matemáticos (Bergstrom et al., 2012; Bull, Housh, Johnson, \& Perry, 2000; Hill, 1993). Os modelos de dois parâmetros (lineares - equações 1 e 2 e hiperbólico - equação 3) têm sido comumente adotados para estimar a PC e a capacidade de trabalho anaeróbio (CTA) (Bull et al., 2000; Dekerle, Brickley, Hammond, Pringle, \& Carter, 2006).

$$
\begin{aligned}
& \mathrm{P}=(\mathrm{CTA} / \mathrm{tlim})+\mathrm{PC} \\
& \text { Trabalho }=(\mathrm{PC} \mathrm{x} \text { tlim })+\mathrm{CTA} \\
& \text { tlim }=\mathrm{CTA} /(\mathrm{P}-\mathrm{PC})
\end{aligned}
$$

Onde: tlim = tempo limite, $\mathrm{CTA}=$ capacidade de trabalho anaeróbio, $\mathrm{P}=$ potência e $\mathrm{PC}=$ potência crítica.

De acordo com o modelo da PC, existem apenas dois componentes que fornecem energia para o exercício (i.e., anaeróbio e aeróbio) (Morton, 2006). O metabolismo aeróbio possui grande capacidade energética (i.e., total de energia que pode ser transferida para a ressíntese de adenosina trifosfato - ATP), mas é limitado em relação à sua taxa de produção de energia (i.e., quantidade de energia que pode ser transferida para a ressíntese de ATP por unidade de tempo). No modelo, a PC representa o limite superior de intensidade de exercício onde existe uma sustentável taxa de ressíntese de ATP que é realizada predominantemente pelo metabolismo aeróbio, podendo ser mantida teoricamente por um tempo indefinido (Morton, 2006). De fato, vários estudos com delineamentos transversais (ex., correlações entre a PC e a resposta do lactato ao exercício) (Denadai, Gomide, \& Greco, 2005; Greco, Caritá, Dekerle, \& Denadai, 2012) e longitudinais (efeitos do treino) (Jenkins \& Quigley, 1992) têm confirmado que a PC é sensível a mudanças que podem ocorrer no metabolismo aeróbio em função dos diferentes níveis de aptidão aeróbia. Ainda de acordo com o modelo, o metabolismo anaeróbio apresenta uma alta taxa de produção energética, mas com capacidade de produção de energia reduzida, sendo seus estoques representados pela CTA. Assim, a CTA compreende os estoques limitados de energia, ou seja, as reservas de fosfatos (ATP e creatina fosfato - CP), a quantidade de $\mathrm{O}_{2}$ no sangue e ligado à mioglobina e a glicólise anaeróbia, podendo ser definida como a quantidade constante de trabalho que pode ser realizada acima da PC.

Tem sido descrito que o aquecimento / exercício prévio pode melhorar a performance (i.e., tlim) de um exercício subsequente realizado acima da PC (i.e., domínio severo) (Jones, Wilkerson, Burnley, \& Koppo, 2003). Muitos estudos têm relacionado este aumento do tlim 
a fatores como o aumento da velocidade da condução nervosa, alteração na relação forçavelocidade da contração muscular e/ou aumento do fluxo sanguíneo muscular (Bishop, 2003; Carter et al., 2005; Gerbino, Ward, \& Whipp, 1996). Carter, Grice, Dekerle, Brickley, Hammond, e Pringle (2005) e Burnley, Doust, e Jones (2002) demonstraram que o exercício prévio pode influenciar a cinética do $\mathrm{VO}_{2}$, aumentando a amplitude primária e diminuindo a componente lenta, o que pode reduzir a contribuição anaeróbia no início do exercício. Assim, de acordo com o modelo da PC, a tolerância (tempo de exaustão) ao exercício realizado no domínio severo pode aumentar pela menor taxa de depleção da CTA no início do exercício (Jones, Wilkerson, DiMenna, Fulford, \& Poole, 2008).

O exercício prévio realizado no domínio pesado (i.e., < PC) seguido de uma recuperação suficiente $(>$ 9-10 $\mathrm{min}$ ) pode prolongar o tlim em até $60 \%$ (Bailey, Vanhatalo, Wilkerson, Dimenna, \& Jones, 2009; Carter et al., 2005; Jones et al., 2003). Em contraste, o exercício prévio realizado no domínio severo (carga para induzir a exaustão entre 6-8 min) seguido por recuperações mais curtas (2-6 min) (Ferguson et al., 2007, 2010) ou mesmo mais longas (15 min) (Ferguson et al., 2010) pode reduzir o tlim, refletindo na redução da CTA. Entretanto, o exercício severo realizado em menor intensidade $(70 \%$ da diferença da carga do limiar ventilatório - $\mathrm{LV}$ e do $\mathrm{VO}_{2}$ pico - $\mathrm{VO}_{2}$ pico, tlim $\sim 15-20 \mathrm{~min}$ ) e com recuperação suficiente (> 6 min) aumenta em até $15-30 \%$ o tlim no domínio severo (Bailey et al., 2009). Assim, para o exercício prévio realizado no domínio severo, parece existir uma relação ótima entre a intensidade e a duração da recuperação, para que a tolerância ao exercício possa ser aumentada. Esta combinação provavelmente otimiza o balanço entre os efeitos positivos do exercício prévio na cinética do $\mathrm{VO}_{2}$ e o tempo suficiente de recuperação da homeostase muscular (restauração de $\mathrm{CP}$ e íons $\mathrm{H}^{+}$). Além disso, tem sido verificado um aumento da eletromiografia integrada (iEMG) nos dois primeiros minutos de exercício severo que é realizado após o exercício prévio, sugerindo uma mudança no padrão de recrutamento muscular (Burnley et al., 2002). Esta mudança no início do exercício permite que a demanda metabólica por fibra muscular seja reduzida e que o recrutamento adicional de fibras ao longo do exercício severo (i.e., acima de 2 min de exercício), possa ser menor (Bailey et al., 2009). O padrão de recrutamento muscular tem implicações no desenvolvimento da componente lenta do $\mathrm{VO}_{2}$, a qual também se tem mostrado menor após o exercício prévio (Bailey et al., 2009). Menor componente lenta do $\mathrm{VO}_{2}$ pode explicar o aumento da tolerância no exercício realizado no domínio severo (Jones et al., 2011).

Deste modo, o objetivo deste estudo foi investigar o efeito do exercício prévio severo na estimativa da PC e da CTA em indivíduos ativos. Baseado nos estudos citados anteriormente (Bailey et al., 2009), foi colocada a hipótese de que o exercício prévio severo, que permite exaustão por volta de $15-20 \mathrm{~min}$, e tempo de recuperação adequado ( $>6 \mathrm{~min}$ ), pode aumentar o tlim e, consequentemente, a PC, sem modificar a CTA.

\section{MÉTODO}

\section{Amostra}

Participaram neste estudo sete indivíduos do sexo masculino, ativos mas não treinados em qualquer tipo de treinamento regular, específico do ciclismo (idade: $21.85 \pm 1.21$ anos; massa corporal: $80.58 \pm 13.86 \mathrm{~kg}$; estatura: $175.28 \pm 5.34 \mathrm{~cm} ; \mathrm{VO}_{2} \max : 3527.28 \pm 612.42$ $\left.\mathrm{ml} . \mathrm{min}^{-1}\right)$. Todos os indivíduos foram informados sobre os procedimentos do experimento e suas implicações, assinando um termo de consentimento livre e esclarecido para participação deste estudo. O protocolo deste estudo foi aprovado pelo comitê de ética local em pesquisa da universidade onde o estudo foi conduzido e foi realizado de acordo com a declaração de Helsinki. 


\section{Instrumentos}

Os testes foram realizados em um cicloergômetro de frenagem eletromagnética (Excalibur Sport, Lode, BV, Croningen, Holanda). O $\mathrm{VO}_{2}$ foi mensurado respiração a respiração durante todos os testes a partir do gás expirado (QuarkPFTergo, Cosmed, Roma, Itália). A análise da concentração de lactato sanguínea ([La]) foi feita por meio de um analisador eletroquímico (YSI 2300 STAT, Yellow Spring, Ohio, EUA). A frequência cardíaca (FC) foi mensurada por meio da utilização de monitor cardíaco Polar (S810, Polar Electro, Finland).

\section{Procedimentos}

Foram realizados sete testes em dias diferentes, dentro de um período de 2 a 3 semanas. Foi observado pelo menos 48 horas de intervalo entre cada sessão experimental. Inicialmente os sujeitos realizaram um teste progressivo para determinação do $\mathrm{LV}$, do $\mathrm{VO}_{2} \mathrm{max}$ e da sua respetiva intensidade $\left(\mathrm{IVO}_{2} \max \right)$. A seguir foram realizados seis testes preditivos de carga constante com e sem a realização de um exercício prévio severo nas intensidades de 95, 100 e $110 \% \mathrm{IVO}_{2}$ max em ordem aleatória para determinação da PC e da CTA. Todos os testes foram realizados em dias diferentes, em um laboratório com condições ambientais controladas (temperatura $21-23^{\circ} \mathrm{C}$, humidade relativa do ar - 40 - 50\%) e no mesmo horário do dia ( $\pm 2 \mathrm{~h})$. Os sujeitos foram instruídos a não praticar exercícios exaustivos no dia anterior ao da avaliação, não ingerir bebidas contendo cafeína e álcool nas 24 horas que antecederam os testes e a comparecerem alimentados e hidratados no dia do teste. Todos os voluntários relataram ter cumprido estas orientações.

\section{Protocolo incremental}

Os sujeitos realizaram um teste progressivo para determinação do $\mathrm{LV}, \mathrm{VO}_{2} \max$ e $\mathrm{IVO}_{2} \max$ com intensidade inicial de $35 \mathrm{~W}$ e incrementos de $35 \mathrm{~W}$ a cada 3 minutos, com frequência de pedalada mantida a $70 \mathrm{rpm}$ até a exaustão voluntária (Caputo \& Denadai, 2008). No final de cada estágio foram coletados, sem pausa, a
FC e $25 \mu \mathrm{l}$ de sangue do lóbulo da orelha. A calibração do sistema de análise das concentrações de oxigênio $\left(\mathrm{O}_{2}\right)$ e gás carbônico $\left(\mathrm{CO}_{2}\right)$ foi realizada antes de cada teste, usando ar ambiente e um gás com concentrações conhecidas de $\mathrm{O}_{2}$ e $\mathrm{CO}_{2}$ de acordo com as instruções do fabricante (Cosmed, 2003). A turbina do analisador foi calibrada através de uma seringa de três litros (Cosmed, Roma, Itália). Os dados de $\mathrm{VO}_{2}$ foram suavizados e obtidas as médias de $15 \mathrm{~s}$, sendo que o mais alto valor neste intervalo foi considerado como $\mathrm{O} \mathrm{VO}_{2}$ max. Todos os sujeitos preencheram no mínimo dois dos três critérios para $\left.\mathrm{o} \mathrm{VO}_{2} \max : 1\right)$ Razão de trocas gasosas $(\mathrm{R}) \geq 1.1$; 2) Frequência cardíaca máxima no mínimo igual $90 \%$ da máxima prevista pela idade; 3) concentração pico de lactato maior que $8 \mathrm{mM}$ (Taylor, Buskirk, \& Henschel, 1955). O LV foi estimado conforme as recomendações de Beaver, Wasserman, e Whipp (1986), no qual o LV foi examinado visualmente, usando as respostas dos parâmetros equivalentes ventilatórios do $\mathrm{CO}_{2}\left(\mathrm{VE} / \mathrm{CO}_{2}\right)$ e do $\mathrm{O}_{2}$ (VE/ $\mathrm{O}_{2}$ ) e das frações expiradas de $\mathrm{CO}_{2}$ $\left(\mathrm{PETCO}_{2}\right)$ e de $\left.\mathrm{O}_{2}(\mathrm{PET} \mathrm{O})_{2}\right)$. Os critérios para determinar o LV foram: aumento na curva da relação $\mathrm{VE} / \mathrm{VO}_{2}$ e na $\mathrm{P}_{\mathrm{ET}} \mathrm{O}_{2}$, sem alteração da relação $\mathrm{VE} / \mathrm{VCO}_{2}$ e na $\mathrm{P}_{\mathrm{ET}} \mathrm{CO}_{2}$. O LV foi detetado por dois observadores independentes. No caso de discordância entre os avaliadores (1 voluntário), um terceiro pesquisador foi consultado. A $\mathrm{IVO}_{2} \max$ foi considerada como sendo a menor intensidade de exercício na qual ocorreu o $\mathrm{VO}_{2} \max$ (Billat, Hill, Pinoteau, Petit, \& Koralsztein, 1996).

\section{Protocolo para determinação da PC e CTA nas} condições controle (CON) e com exercício prévio severo (EPS)

Os voluntários foram submetidos a 6 testes de carga constante com (EPS) e sem (CON) a realização de um exercício prévio severo, nas intensidades de 95,100 e $110 \% \mathrm{IVO}_{2} \max$. Todos os testes foram realizados em ordem aleatória e em dias diferentes (Caputo \& Denadai, 2008). Na condição CON os voluntários pedalaram a $20 \mathrm{~W}$ por $3 \mathrm{~min}$, 
posteriormente, a intensidade foi ajustada para 95, 100 ou $110 \% \mathrm{IVO}_{2}$ max e os voluntários pedalaram até a exaustão voluntária ou até que não pudessem manter a intensidade estipulada (cadência < $70 \mathrm{rpm}$ ) (Figura 1). Na condição EPS, os sujeitos pedalaram a $20 \mathrm{~W}$ por $3 \mathrm{~min}$, posteriormente realizaram um EPS por $6 \mathrm{~min}$ a $70 \% \Delta$ (70\% da diferença entre o LV e a $\mathrm{IVO}_{2} \max$ ), seguidos por $6 \mathrm{~min}$ a $20 \mathrm{~W}$ (Burnley, Doust, Carter, \& Jones, 2001). Foi realizada uma coleta de sangue do lóbulo da orelha no final do 60 min do EPS e no final do 6o min de recuperação para dosagem da [La]. Posteriormente, a intensidade foi ajustada para 95, 100 ou $110 \% \mathrm{IVO}_{2} \max$ e os indivíduos pedalaram até a exaustão voluntária ou até que não pudessem manter a intensidade estipulada (cadência < $70 \mathrm{rpm}$ ) (Figura 1). As intensidades das cargas preditivas foram escolhidas para se atingir a exaustão entre 3 15 min, aproximadamente (di Prampero, 1999; Hill, 1993). O tlim foi considerado como o tempo total de esforço mantido na carga preditiva e expresso em segundos (Hill, 1993). Durante todo o teste o indivíduo foi encorajado a realizar o esforço máximo, não recebendo, entretanto nenhuma informação sobre a duração do teste. A FC foi monitorada durante todo o teste e ao final do mesmo foi realizada uma coleta de sangue do lóbulo da orelha para dosagem da [La]. $\mathrm{O} \quad \mathrm{VO}_{2}$ foi mensurado respiração a respiração durante todo o protocolo a partir do gás expirado, sendo os dados reduzidos às médias de $15 \mathrm{~s}$. $\mathrm{O} \mathrm{VO}_{2}$ pico foi considerado o maior valor médio de $15 \mathrm{~s}$ analisado durante o teste de carga constante.

Os valores individuais de potência e tlim obtidos durante os testes de carga constante foram ajustados a partir dos três modelos de dois parâmetros $\left[\mathrm{P}=\mathrm{CTA} / \mathrm{t}_{\mathrm{lim}}\right)+\mathrm{PC}$; $\mathrm{t}_{\text {lim }}=$ CTA/(P-PC); P = PC.t lim $_{\text {lim }}+$ CTA] para estimar a PC e a CTA (Bull et al., 2000). A PC e a CTA determinadas pelas três equações foram comparadas e o modelo com o menor erro padrão da estimativa (Hill \& Smith, 1994), foi utilizado para as comparações nas diferentes condições experimentais.

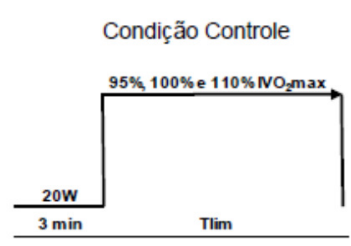

Condição Exercício Prévio Severo

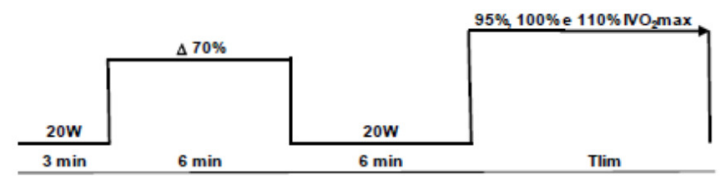

Figura 1. Esquema representativo do delineamento experimental. No topo da figura, a condição controle $(\mathrm{CON})$, e na parte inferior a condição com a realização do exercício prévio severo (EPS).

Determinação dos parâmetros da cinética do consumo de oxigênio

Nos testes de carga constante realizados a 95, 100 e $110 \% \mathrm{IVO}_{2}$ max os valores do $\mathrm{VO}_{2}$ medido respiração-a-respiração foram filtrados e interpolados para obter-se um valor a cada segundo. Os dados dos primeiros $20 \mathrm{~s}$ de exercício foram removidos da análise.

Para a intensidade de $95 \% \mathrm{IVO}_{2} \max$, a análise da cinética do $\mathrm{VO}_{2}$ foi feita por meio de um modelo bi-exponencial (Equação 4).

$V O_{2}(t)=V O_{2 b}+A_{1}\left[1-\ell^{-\left(t-T D_{1} / \tau_{1}\right)}\right]+A_{2}\left[1-\ell^{-\left(t-T D_{2} / \tau_{2}\right)}\right]$

Onde: $\mathrm{VO}_{2}(\mathrm{t})$ - consumo de oxigênio no tempo t; $\mathrm{VO}_{2 \mathrm{~b}}-\mathrm{VO}_{2}$ de base representa $\mathrm{O} \mathrm{VO}_{2}$ médio dos últimos $60 \mathrm{~s}$ antes do início do exercício; $\mathrm{A}_{1}$ - amplitude da resposta primária do $\mathrm{VO}_{2}$ (diferença entre o $\mathrm{VO}_{2}$ assíntota e o $\mathrm{VO}_{2}$ base); o $\mathrm{TD}_{1}$ é o tempo de atraso da resposta primária do $\mathrm{VO}_{2}$; o $\tau_{1}$ é a constante de tempo da resposta primária do $\mathrm{VO}_{2}$ (definida como o tempo necessário para atingir $63 \%$ de $\mathrm{A}$ ); o $\mathrm{A}_{2}$ é a amplitude da sua resposta secundária; o $\mathrm{TD}_{2}$ é o tempo de atraso da resposta secundária do $\mathrm{VO}_{2} ; \mathrm{o}$ $\tau_{2}$ é a constante de tempo da resposta secundária.

$\mathrm{O}$ incremento relevante do $\mathrm{VO}_{2}$ corresponde à amplitude da fase I $\left(\mathrm{A}_{1}{ }^{\prime}\right)$, que reflete de forma mais próxima a cinética da troca gasosa em nível muscular, foi calculado da seguinte forma: 


$$
A_{1}^{\prime}=A_{1}\left(1-\ell^{\left(-\left(T D_{2}-T D_{1}\right) / \tau_{1}\right)}\right)
$$

Como o valor da assíntota $\left(\mathrm{A}_{2}\right)$ pode representar um valor maior do que o real no final do exercício, o valor do componente exponencial lento do $\mathrm{VO}_{2}$ no final do exercício foi definido como $\mathrm{A}_{2}^{\prime}$ :

$$
A_{2}^{\prime}=A_{2}\left(1-\ell^{\left(-\left(E D-T D_{2}\right) / \tau_{2}\right)}\right)
$$

Onde: ED é a duração do exercício.

Para as intensidades de 100 e $110 \% \mathrm{IVO}_{2} \max$, os valores de $\mathrm{VO}_{2}$ foram plotados por meio de um modelo monoexponencial por meio da seguinte equação:

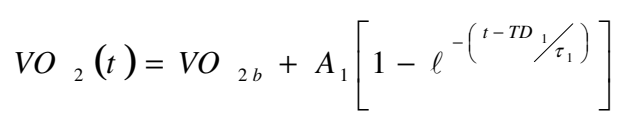

Onde: $\mathrm{VO}_{2}(\mathrm{t})$ - consumo de oxigênio no tempo t; $\mathrm{VO}_{2 \mathrm{~b}}-\mathrm{VO}_{2}$ de base; A - amplitude da resposta pri- mária do $\mathrm{VO}_{2}$; o TD é o tempo de atraso da resposta primária do $\mathrm{VO}_{2} ;$ o $\tau$ é a constante de tempo da resposta primária do $\mathrm{VO}_{2}$.

\section{Análise Estatística}

Foram calculadas as médias \pm DP dos dados obtidos. A existência de normalidade dos dados foi verificada por meio do teste de Shapiro-Wilk. A comparação entre as diferentes condições (CON vs. EPS) foi realizada por meio do teste $t$ Student para dados pareados. O tamanho do efeito (Score d) foi calculado de acordo com Cohen (1988). Para a análise da cinética do $\mathrm{VO}_{2}$ foi utilizado o software Microcal Origin 6.0 (Northampton, MA, EUA). Em todos os testes foi adotado um nível de significância de $p<0.05$. Todas as análises estatísticas foram realizadas utilizando o programa estatístico SPSS 18.0.

\section{RESULTADOS}

As variáveis obtidas no teste incremental estão apresentadas na Tabela 1.

\begin{tabular}{|c|c|}
\hline & Média \pm DP \\
\hline $\mathrm{IVO}_{2} \max (\mathrm{W})$ & $271.1 \pm 40.6$ \\
\hline $\mathrm{VO}_{2} \max \left(\mathrm{ml} \cdot \mathrm{min}^{-1}\right)$ & $3527.3 \pm 612.4$ \\
\hline $\mathrm{VO}_{2} \max \left(\mathrm{ml} \cdot \mathrm{kg}^{-1} \cdot \mathrm{min}^{-1}\right)$ & $44.0 \pm 5.7$ \\
\hline LV (W) & $125.0 \pm 18.7$ \\
\hline $\mathrm{LV} \%\left(\% \mathrm{IVO}_{2} \max \right)$ & $46.4 \pm 5.8$ \\
\hline FCmax (bpm) & $182 \pm 5$ \\
\hline [La]pico (mM) & $11.6 \pm 1.3$ \\
\hline $\mathbf{R}$ & $1.21 \pm 0.12$ \\
\hline
\end{tabular}

Tabela 1

Valores médios \pm DP das variáveis obtidas no protocolo incremental $(N=7)$

A [La] obtida antes da sessão principal de exercício foi significativamente maior na condição EPS do que na condição CON (6.51 \pm 0.52 e $1.23 \pm 0.24 \mathrm{mM}$, respetivamente) $(p<$ $0.001, d=13.92)$. A intensidade do EPS (i.e.,
$70 \% \Delta$ ) foi de $229.75 \pm 33.37 \mathrm{~W}$, sendo significativamente maior do que a PC (199.71 \pm $27.08 \mathrm{~W})(p=0.007, d=0.99)$. O thim estimado para o exercício a $70 \% \Delta$ foi de $23.10 \pm$ $18.56 \mathrm{~min}$. 
A Tabela 2 apresenta os valores do tlim, FC e [La] correspondentes a 95, 100 e $110 \% \mathrm{IVO}_{2}$ max com (EPS) e sem (CON) a realização do exercício prévio severo. O tlim nas intensidades de 100 e $110 \% \mathrm{IVO}_{2}$ max foi significativamente superior na condição $\mathrm{CON}$ do que na condição EPS ( $p=0.02, d=0.46$ e $p=$ $0.02, d=0.60$, respetivamente).

A tabela 3 apresenta os valores médios \pm DP dos parâmetros da cinética do $\mathrm{VO}_{2}$ nos exercícios de carga constante com (EPS) e sem $(\mathrm{CON})$ a realização do exercício prévio severo.

Tabela 2

Valores médios $\pm D P$ das variáveis obtidas a 95, 100 e 110\% da intensidade correspondente ao consumo máximo de oxigênio (IVO $\mathrm{IVax}_{2}$ ) nas condiçôes controle (CON) e exercício prévio severo (EPS). $N=7$

\begin{tabular}{|c|c|c|c|}
\hline & & $\mathrm{CON}$ & EPS \\
\hline \multirow{3}{*}{$95 \% \mathrm{IVO}_{2} \max$} & $\mathrm{t} \lim (\mathrm{s})$ & $501 \pm 140$ & $473 \pm 99$ \\
\hline & FC (bpm) & $182 \pm 6$ & $184 \pm 6$ \\
\hline & {$[\mathrm{La}]_{\text {final }}(\mathrm{mM})$} & $10 \pm 1.4$ & $11.1 \pm 2.7$ \\
\hline \multirow{3}{*}{$100 \% \mathrm{IVO}_{2} \max$} & $\operatorname{tlim}(s)$ & $381 \pm 103$ & $334 \pm 101^{*}$ \\
\hline & FC (bpm) & $180 \pm 7$ & $185 \pm 8$ \\
\hline & {$[\mathrm{La}]_{\text {final }}(\mathrm{mM})$} & $9.9 \pm 2.8$ & $10.5 \pm 1.1$ \\
\hline \multirow{3}{*}{$110 \% \mathrm{IVO}_{2} \max$} & $\operatorname{tlim}(s)$ & $267 \pm 66$ & $227 \pm 68^{*}$ \\
\hline & FC (bpm) & $181 \pm 8$ & $187 \pm 8$ \\
\hline & {$[\mathrm{La}]_{\text {final }}(\mathrm{mM})$} & $8.9 \pm 2.4$ & $11.6 \pm 2.7$ \\
\hline
\end{tabular}

Tabela 3

Valores médios $\pm D P$ dos parâmetros da cinética do consumo de oxigênio $\left(\mathrm{VO}_{2}\right)$ nos exercícios de carga constante com (EPS) e sem (CON) a realização do exercício prévio severo. $N=7$

\begin{tabular}{|c|c|c|c|}
\hline & & $\mathrm{CON}$ & EPS \\
\hline \multirow{8}{*}{$95 \% \mathrm{IVO}_{2} \max$} & $\mathrm{VO}_{2}$ base $\left(\mathrm{ml} . \mathrm{min}^{-1}\right)$ & $844,3 \pm 55,7$ & $821,4 \pm 85,7$ \\
\hline & TD1 (s) & $1,81 \pm 2,2$ & $3,39 \pm 4,3$ \\
\hline & $\tau 1(\mathrm{~s})$ & $20,6 \pm 6,5$ & $20,5 \pm 10,3$ \\
\hline & $A_{1}^{\prime}\left(\mathrm{ml} \cdot \mathrm{min}^{-1}\right)$ & $2145,3 \pm 501$ & $2271,4 \pm 344,3$ \\
\hline & $\mathrm{TD}_{2}(\mathrm{~s})$ & $94 \pm 45$ & $107,6 \pm 30,8$ \\
\hline & $\tau_{2}(s)$ & $97,6 \pm 76,1$ & $89,1 \pm 71,5$ \\
\hline & $\mathrm{A}_{2}^{\prime}\left(\mathrm{ml} \cdot \mathrm{min}^{-1}\right)$ & $578,3 \pm 301,7$ & $352,3 \pm 142,5$ \\
\hline & $\mathrm{VO}_{2}$ pico $\left(\mathrm{ml} \cdot \mathrm{min}^{-1}\right)$ & $3608,7 \pm 596,9$ & $3596,9 \pm 549,1$ \\
\hline \multirow{5}{*}{$100 \% \mathrm{IVO}_{2} \max$} & $\mathrm{VO}_{2}$ base $\left(\mathrm{ml} \cdot \mathrm{min}^{-1}\right)$ & $851,1 \pm 41,2$ & $823,1 \pm 34,4$ \\
\hline & $\tau(\mathrm{s})$ & $46,5 \pm 16$ & $34,1 \pm 15,9^{*}$ \\
\hline & $\mathrm{A}_{1}\left(\mathrm{ml} \cdot \mathrm{min}^{-1}\right)$ & $2631,5 \pm 479,6$ & $2733,8 \pm 651,1$ \\
\hline & TD (s) & $10,7 \pm 8$ & $7,7 \pm 7,6$ \\
\hline & $\mathrm{VO}_{2}$ pico $\left(\mathrm{ml} \cdot \mathrm{min}^{-1}\right)$ & $3665,6 \pm 753,0$ & $3403,4 \pm 623,6$ \\
\hline \multirow{5}{*}{$110 \% \mathrm{IVO}_{2} \max$} & $\mathrm{VO}_{2}$ base $\left(\mathrm{ml} \cdot \mathrm{min}^{-1}\right)$ & $845,9 \pm 78,5$ & $834,4 \pm 73$ \\
\hline & $\tau(\mathrm{s})$ & $37,3 \pm 10,3$ & $23,6 \pm 5,6^{*}$ \\
\hline & $A_{1}\left(\mathrm{ml} \cdot \mathrm{min}^{-1}\right)$ & $2773,4 \pm 657,6$ & $2601,3 \pm 591,5$ \\
\hline & TD (s) & $4,4 \pm 3,4$ & $2,1 \pm 2,6$ \\
\hline & $\mathrm{VO}_{2}$ pico $\left(\mathrm{ml} \cdot \mathrm{min}^{-1}\right)$ & $3734,9 \pm 596,6$ & $3547,2 \pm 542,7$ \\
\hline
\end{tabular}

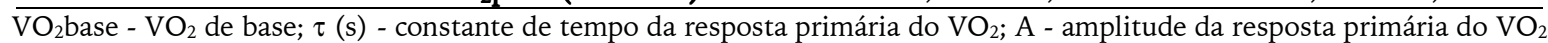
$\left(\mathrm{ml} \cdot \mathrm{min}^{-1}\right)$; TD (s) - tempo de atraso da resposta primária do $\mathrm{VO}_{2}$; $\mathrm{VO}_{2}$ pico $\left(\mathrm{ml} \cdot \mathrm{min}^{-1}\right)$ - consumo de oxigênio pico. ${ }^{*} \mathrm{p}<$ 0.05 em relação à condição $\mathrm{CON}$. 
$\mathrm{O} \tau$ foi significativamente menor após o exercício prévio nas intensidades de $100 \% \mathrm{IVO}_{2} \max (p=0.04, d=0.78) \mathrm{e}$ $110 \% \mathrm{IVO}_{2} \max (p=0.003, d=1.65) . \mathrm{O}$ $\mathrm{VO}_{2}$ pico atingido ao final dos testes preditivos nas condições CON e EPS não foi significativamente diferente do $\mathrm{VO}_{2} \max (p>0.05)$.
A PC na condição CON $(199.71 \pm 27.08 \mathrm{~W}$; $74.12 \pm 7.56 \% \mathrm{IVO}_{2} \max$ ) não foi significativamente diferente em relação à condição EPS $\left(211.71 \pm 29.92 \mathrm{~W} ; 78.33 \pm 6.87 \% \mathrm{IVO}_{2} \max \right)$ $(p>0.05)$. Da mesma forma, a CTA na condição CON (23.46 $\pm 10.76 \mathrm{~kJ})$ não foi significativamente diferente relativamente à condição EPS $(17.51 \pm 8.44 \mathrm{~kJ})(p>0.05)$ (Figura 2).

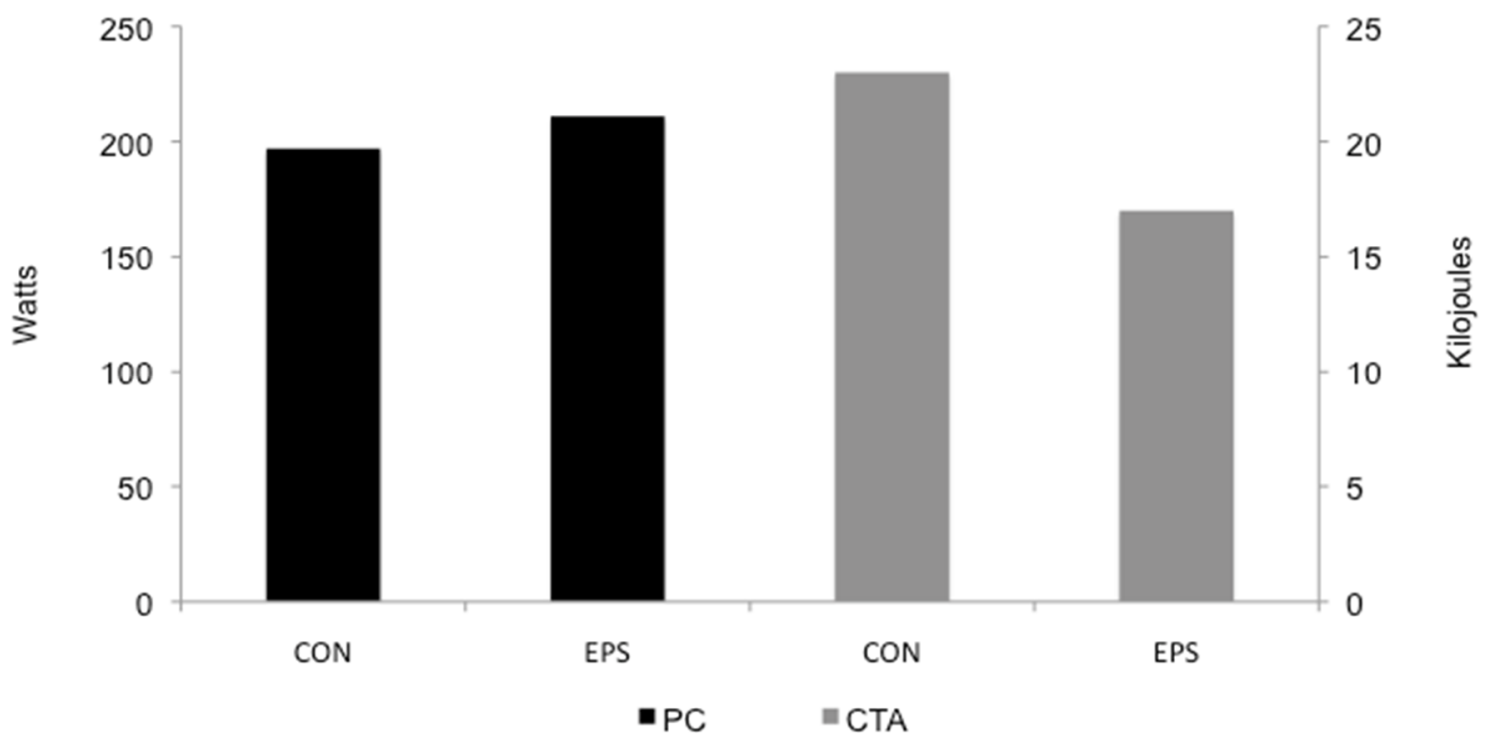

Figura 2. Valores médios da potência critica (PC) e da capacidade de trabalho anaeróbio (CTA) com (EPS) e sem $(\mathrm{CON})$ a realização do exercício prévio severo. $\mathrm{N}=7$

\section{DISCUSSÃO}

O objetivo deste estudo foi analisar o efeito do EPS nos parâmetros da relação potência tempo em indivíduos ativos. Nosso principal achado foi que a PC e CTA não são modificados pelo EPS durante o ciclismo. Esse dado contraria, pelo menos em parte, a nossa hipótese inicial de que em indivíduos ativos o EPS poderia influenciar a estimativa da PC sem modificar a CTA. Esse resultado é semelhante ao obtido por Burnley, Davison, e Baker (2011) que não encontraram diferenças na estimativa da PC, após 10 min da realização de um exercício prévio severo realizado durante $6 \mathrm{~min}$ em uma carga estimada que levaria à exaustão em $8 \mathrm{~min}$.
Um especto que inicialmente deve ser analisado nesse estudo é a validade do protocolo utilizado para determinar a PC, já que este índice é protocolo-dependente (Bishop, Jenkins, \& Howard, 1998; Calis \& Denadai, 2000). Diversos estudos mostram que a PC é dependente da duração (tlim) que as cargas selecionadas permitem (isto é, quanto maior a duração das cargas utilizadas na predição menor a PC). Neste sentido, di Prampero (1999) sugere que as cargas selecionadas para a determinação da PC devem permitir um tlim entre 2 e 15 min e que o $\mathrm{VO}_{2} \max$ seja atingido ao final do exercício. No presente estudo, embora o tlim nas cargas mais altas (100\% e $\left.110 \% \mathrm{IVO}_{2 \max }\right)$ tenha sido reduzido pelo EPS, o mesmo está dentro do sugerido pela literatura 
( 4-9 min). Além disso, o $\mathrm{VO}_{2}$ pico atingido em todos os testes preditivos das condições CON e EPS não foi, significativamente, diferente do $\mathrm{VO}_{2} \max$. Diversos estudos têm verificado que a cinética do $\mathrm{VO}_{2}$ é alterada durante o exercício severo realizado após o exercício pesado (Bailey et al., 2009; Burnley, Doust, \& Jones, 2002) e severo (Burnley, Davison, \& Baker, 2011; Burnley, Doust, \& Jones, 2005). Nestes estudos têm-se encontrado que a amplitude do componente primário pode ser aumentada, com (Burnley et al., 2011) ou sem (Burnley et al., 2005) modificações na taxa de ajuste do $\mathrm{VO}_{2}$ (i.e., $\tau$ ). Estas modificações na cinética do $\mathrm{VO}_{2}$ nos momentos iniciais do exercício, têm sido associadas à melhoria da tolerância (tlim) durante o esforço severo, já que haveria redução na taxa de utilização das reservas anaeróbias (i.e., CTA). De fato, o tlim durante o exercício severo parece ser influenciado pela taxa de depleção da reservas de creatina fósforo (CP) e/ou pelo acúmulo de metabolitos (i.e., $\mathrm{H}^{+}$, Pi) (Miura, Sato, Sato, Whipp, \& Fukuba, 2000). Assim, a aceleração da cinética do $\mathrm{VO}_{2}$ no início do exercício diminui a taxa de utilização de CP e o acúmulo de metabolitos, podendo modificar o tlim no exercício severo. No nosso estudo, a cinética do $\mathrm{VO}_{2}$ (i.e., $\tau$ ) foi acelerada durante as cargas mais altas $\left(100 \%\right.$ e $\left.110 \% \mathrm{IVO}_{2} \max \right)$. Entretanto, o tlim nestas intensidades foi significativamente menor após o EPS. O exercício severo, diferente do exercício pesado, leva a uma depleção progressiva de $\mathrm{CP}$ e o acúmulo de metabolitos (Jones et al., 2008), sendo que a recuperação da CTA necessita de períodos de tempo relativamente longos $\left(t_{1 / 2} \sim 234 \mathrm{~s}\right)$ (Ferguson et al., 2010). Portanto, a aceleração da cinética do $\mathrm{VO}_{2}$ não parecer ser suficiente para contrabalançar os menores estoques de $\mathrm{CP}$ e/ou maior acúmulo de metabolitos determinados pelo EPS, levando a uma diminuição do tlim nas intensidades mais elevadas.

Interessantemente, Bailey, Vanhatalo, Wilkerson, Dimenna, e Jones (2009) verificaram recentemente que o tlim durante o exercício severo $\left(80 \% \Delta, \sim 80 \% \mathrm{IVO}_{2} \max \right)$ aumentou em até $15-30 \%$ após EPS $(70 \% \Delta)$ com recuperação suficiente (> $6 \mathrm{~min}$ ), não aumentando entretanto após o exercício prévio pesado $(40 \% \Delta)$, independente do período de recuperação. Em nosso estudo, o tlim do exercício severo de menor intensidade (i.e., $95 \% \mathrm{IVO}_{2} \mathrm{max}$ ) não foi modificado pelo EPS. Se somarmos a estes dados que o exercício prévio pesado $(<\mathrm{PC})$ aumenta o tlim do exercício severo de intensidade máxima $\left(100 \% \mathrm{IVO}_{2} \max \right)$ e supramáxima (> 100\% $\left.\mathrm{IVO}_{2} \max \right)$ (Jones et al., 2003), é possível colocar a hipótese sobre as possíveis relações entre exercício prévio e a tolerância no domínio severo. Para os exercícios no domínio severo de intensidade submáxima (< $\left.95 \% \mathrm{IVO}_{2} \mathrm{max}\right)$, a tolerância pode ser aumentada quando o exercício prévio é severo e a recuperação é suficiente (> $6 \mathrm{~min}$ ). Nestas intensidades, a taxa de depleção da CTA é mais lenta e os efeitos do exercício prévio severo parecem otimizar mais as alterações que aumentam a tolerância ao exercício (i.e., aumento da amplitude e/ou da cinética do $\mathrm{VO}_{2}$ ). Já nas intensidades maiores $\left(\geq 100 \% \mathrm{IVO}_{2} \max \right)$, onde a taxa de depleção da CTA é proporcionalmente maior e portanto, a tolerância ao esforço mais dependente da CTA, o exercício prévio pesado, por causar menor depleção à CTA, parece aumentar mais a tolerância ao esforço. Assim, parece existir uma relação ótima entre os efeitos da intensidade do exercício prévio (i.e., menor ou maior utilização da CTA), tempo de recuperação (i.e., taxa de restauração da CTA) e a intensidade do exercício realizado subsequentemente (i.e., menor ou maior utilização da CTA).

No presente estudo, o EPS não modificou significativamente a PC, sendo consistente com evidências prévias (Burnley et al., 2011) de que a tolerância no exercício severo não é alterada por modificações da PC. Do mesmo modo, a CTA não foi estatisticamente diferente após o EPS. Entretanto, este dado deve ser analisado com ressalvas, já que a nossa amostra é pequena $(\mathrm{N}=7)$, o que pode levar a um erro do tipo II. Assim, não é possível excluir a possibilidade de que o EPS realizado 6 min antes do exercí- 
cio severo, possa modificar a tolerância ao exercício ao diminuir a CTA.

\section{CONCLUSÕES}

Com base nesses resultados pode-se concluir que o EPS acelera a cinética do $\mathrm{VO}_{2}$ durante as cargas mais altas $(100 \%$ e $\left.110 \% \mathrm{IVO}_{2} \max \right)$. Entretanto, a tolerância ao esforço nestas cargas é diminuída após $6 \mathrm{~min}$ de recuperação. Isto não foi acompanhado por modificações significativas da PC e CTA. Assim, alterações da cinética do $\mathrm{VO}_{2}$ nem sempre produzem aumento da tolerância ao esforço realizado acima da $\mathrm{PC}$.

\section{Agradecimentos:}

Nada a declarar.

\section{Conflito de Interesses:}

Nada a declarar.

\section{Financiamento:}

Nada a declarar.

\section{REFERÊNCIAS}

Bailey, S. J., Vanhatalo, A., Wilkerson, D. P., Dimenna, F. J., \& Jones, A. M. (2009). Optimizing the "priming" effect: influence of prior exercise intensity and recovery duration on $\mathrm{O} 2$ uptake kinetics and severe-intensity exercise tolerance. Journal of Applied Physiology, 107(6), 1743-1756. http://doi.org/10.1152/japplphysiol.00810.20 09

Beaver, W. L., Wasserman, K., \& Whipp, B. J. (1986). A new method for detecting anaerobic threshold by gas exchange. Journal of Applied Physiology, 60(6), 2020-2027.

Bergstrom, H. C., Housh, T. J., Zuniga, J. M., Camic, C. L., Traylor, D. A., Schmidt, R. J., \& Johnson, G. O. (2012). A new single work bout test to estimate critical power and anaerobic work capacity. Journal of Strength and Conditioning Research, 26(3), 656-663. http://doi.org/10.1519/JSC.0b013e31822b730 4

Billat, V. L., Hill, D. W., Pinoteau, J., Petit, B., \& Koralsztein, J. P. (1996). Effect of protocol on determination of velocity at VO2 max and on its time to exhaustion. Archives of Physiology and Biochemistry, 104(3), 313-321. http://doi.org/10.1076/apab.104.3.313.12908

Bishop, D. (2003). Warm up I: potential mechanisms and the effects of passive warm up on exercise performance. Sports Medicine, 33(6), 439-454.

Bishop, D., Jenkins, D. G., \& Howard, A. (1998). The critical power function is dependent on the duration of the predictive exercise tests chosen. International Journal of Sports Medicine, 19(2), 125-129. http://doi.org/10.1055/s-2007-971894

Bull, A. J., Housh, T. J., Johnson, G. O., \& Perry, S. R. (2000). Effect of mathematical modeling on the estimation of critical power. Medicine and Science in Sports and Exercise, 32(2), 526530.

Burnley, M., Davison, G., \& Baker, J. R. (2011). Effects of priming exercise on $\mathrm{VO} 2$ kinetics and the power-duration relationship. Medicine and Science in Sports and Exercise, 43(11), 2171-2179. http://doi.org/10.1249/MSS.0b013e31821ff26 d

Burnley, M., Doust, J. H., Carter, H., \& Jones, A. M. (2001). Effects of prior exercise and recovery duration on oxygen uptake kinetics during heavy exercise in humans. Experimental Physiology, 86(3), 417-425.

Burnley, M., Doust, J. H., \& Jones, A. M. (2002). Effects of prior heavy exercise, prior sprint exercise and passive warming on oxygen uptake kinetics during heavy exercise in humans. European Journal of Applied Physiology, 87(4-5), 424-432. http://doi.org/10.1007/s00421-0020647-8

Burnley, M., Doust, J. H., \& Jones, A. M. (2005). Effects of prior warm-up regime on severeintensity cycling performance. Medicine and Science in Sports and Exercise, 37(5), 838845.

Calis, J. F. F., \& Denadai, B. S. (2000). Influência das cargas selecionadas na determinação da potência crítica determinada no ergômetro de braço em dois modelos lineares. Revista Brasileira de Medicina do Esporte, 6(1), 1-4. http://doi.org/10.1590/S151786922000000100002

Caputo, F., \& Denadai, B. S. (2008). The highest intensity and the shortest duration permitting attainment of maximal oxygen uptake during cycling: effects of different methods and aerobic fitness level. European Journal of Applied Physiology, 103(1), 47-57. http://doi.org/10.1007/s00421-008-0670-5

Carter, H., Grice, Y., Dekerle, J., Brickley, G., Hammond, A. J. P., \& Pringle, J. S. M. (2005). Effect of prior exercise above and below critical 
power on exercise to exhaustion. Medicine and Science in Sports and Exercise, 37(5), 775781.

Cohen, J. (1988). Statistical Power Analysis for the behavioral sciences (2. ${ }^{a}$ ed.). Hillsdale, NJ: Lawrence Erlbaum Associates.

Cosmed. (2003). Quark PFT User manual [Manual]. Rome: Cosmed.

Dekerle, J., Brickley, G., Hammond, A. J. P., Pringle, J. S. M., \& Carter, H. (2006). Validity of the two-parameter model in estimating the anaerobic work capacity. European Journal of Applied Physiology, 96(3), 257-264. http://doi.org/10.1007/s00421-005-0074-8

Denadai, B. S., Gomide, E. B. G., \& Greco, C. C. (2005). The relationship between onset of blood lactate accumulation, critical velocity, and maximal lactate steady state in soccer players. Journal of Strength and Conditioning Research, 19(2), 364-368.

Di Prampero, P. E. (1999). The concept of critical velocity: a brief analysis. European Journal of Applied Physiology and Occupational Physiology, $\quad 80(2), \quad 162-164$. http://doi.org/10.1007/s004210050574

Ferguson, C., Rossiter, H. B., Whipp, B. J., Cathcart, A. J., Murgatroyd, S. R., \& Ward, S. A. (2010). Effect of recovery duration from prior exhaustive exercise on the parameters of the powerduration relationship. Journal of Applied Physiology, 108(4), 866-874. http://doi.org/10.1152/japplphysiol.91425.20 08

Ferguson, C., Whipp, B. J., Cathcart, A. J., Rossiter, H. B., Turner, A. P., \& Ward, S. A. (2007). Effects of prior very-heavy intensity exercise on indices of aerobic function and high-intensity exercise tolerance. Journal of Applied Physiology, 103(3), 812-822. http://doi.org/10.1152/japplphysiol.01410.20 06

Gaesser, G. A., \& Poole, D. C. (1996). The slow component of oxygen uptake kinetics in humans. Exercise and Sport Sciences Reviews, 24(1), 35-70.

Gerbino, A., Ward, S. A., \& Whipp, B. J. (1996). Effects of prior exercise on pulmonary gasexchange kinetics during high-intensity exercise in humans. Journal of Applied Physiology, 80(1), 99-107.

Greco, C. C., Caritá, R. A. C., Dekerle, J., \& Denadai, B. S. (2012). Effect of aerobic training sta- tus on both maximal lactate steady state and critical power. Applied Physiology, Nutrition, and Metabolism, 37(4), 736-743. http://doi.org/10.1139/h2012-047

Hill, D. W. (1993). The critical power concept. A review. Sports Medicine, 16(4), 237-254.

Hill, D. W., \& Smith, J. C. (1994). A method to ensure the accuracy of estimates of anaerobic capacity derived using the critical power concept. The Journal of Sports Medicine and Physical Fitness, 34(1), 23-37.

Jenkins, D. G., \& Quigley, B. M. (1992). Endurance training enhances critical power. Medicine and Science in Sports and Exercise, 24(11), 12831289.

Jones, A. M., Grassi, B., Christensen, P. M., Krustrup, P., Bangsbo, J., \& Poole, D. C. (2011). Slow component of VO2 kinetics: mechanistic bases and practical applications. Medicine and Science in Sports and Exercise, 43(11), 2046-2062. http://doi.org/10.1249/MSS.0b013e31821fcfc 1

Jones, A. M., Wilkerson, D. P., Burnley, M., \& Koppo, K. (2003). Prior heavy exercise enhances performance during subsequent perimaximal exercise. Medicine and Science in Sports and Exercise, 35(12), 2085-2092. http://doi.org/10.1249/01.MSS.0000099108.5 5944.C4

Jones, A. M., Wilkerson, D. P., DiMenna, F., Fulford, J., \& Poole, D. C. (2008). Muscle metabolic responses to exercise above and below the "critical power" assessed using 31P-MRS. American Journal of Physiology. Regulatory, Integrative and Comparative Physiology, 294(2), R585-593. http://doi.org/10.1152/ajpregu.00731.2007

Miura, A., Sato, H., Sato, H., Whipp, B. J., \& Fukuba, Y. (2000). The effect of glycogen depletion on the curvature constant parameter of the power-duration curve for cycle ergometry. Ergonomics, $43(1)$ 133-141. http://doi.org/10.1080/001401300184693

Morton, R. H. (2006). The critical power and related whole-body bioenergetic models. European Journal of Applied Physiology, 96(4), 339-354. http://doi.org/10.1007/s00421-005-0088-2

Taylor, H. L., Buskirk, E., \& Henschel, A. (1955). Maximal oxygen intake as an objective measure of cardio-respiratory performance. Journal of Applied Physiology, 8(1), 73-80. 